\title{
Synthesis and growth of alkali halides films by Pulsed Laser Deposition: An electron microscopy study
}

\author{
Dwight R. Acosta ${ }^{1}$, Elsi Mejía ${ }^{2}$ and Citlali Sánchez ${ }^{2}$ \\ ${ }^{1 .}$ Instituto de Física, Universidad Nacional Autónoma de México. \\ 2. Centro de Ciencias Aplicadas y Desarrollo Tecnológico, Universidad Nacional Autónoma de México.
}

Pulsed laser deposition (PLD) technique has been used the last decades, to produce thin films of an ample diversity of materials [1]. This technique uses high power laser pulses to melt, evaporate and ionize material from a target. The vaporized material consist of neutral atoms, ions, electrons, molecules, etc., which constitute a transient, highly luminous plasma plume that expands rapidly away from the target surface. Film growth occurs on an appropriate placed substrate in which the ablated material is collected. The technique of PLD offers some advantages over other film deposition methods as the possibility to produce films of the exact chemical composition of the target and that relatively high deposition rates can be achieved. There are only few reports of laser ablation and PLD of alkali halides [2-3].There are a large number of variables affecting the properties of the plasma which in turn affect the characteristics of the deposited films, such as laser fluence, background gas pressure and substrate temperature. These parameters allow manipulating the films properties to suit particular applications. In this work, we report the growth of $\mathrm{NaCl}$ films by PLD, using different laser fluences and distances between the target and the substrate.

Alkali halide films were deposited ablating commercial targets of $\mathrm{NaCl}$ with an excimer laser (COMPex 102, Lambda Physik), emitting at $248 \mathrm{~nm}$ and with a pulse duration of $30 \mathrm{~ns}$ (FWHM). Films were deposited at room temperature on $10 \times 10 \mathrm{~mm}^{2}$ corning glass and on 200 mesh TEM grids covered with an amorphous film, using 6,000 laser shots. The substrates were placed in front of the target at distances running from 4 to $10 \mathrm{~cm}$. Deposition was carried out in a stainless steel vacuum chamber evacuated until a base pressure of $1 \times 10^{-6}$ Torr. The laser beam was focused using a plane-convex lens with focal length of $50 \mathrm{~cm}$, leading to fluences from 0.2 to $1 \mathrm{~J} / \mathrm{cm}^{2}$.

$\mathrm{NaCl} \mathrm{HREM}$ micrographs reveal that the terrace configurations frequently observed in SEM micrographs at micrometer level, exists since the initial steps of $\mathrm{NaCl}$ grains synthesis. The advances of research findings suggest that it is possible obtain polycrystalline $\mathrm{NaCl}$ thin films by PLD; the observed phenomena during the films formation have not been previously seen, such as the coalescence and the autoensemble of the nanocrystals as a function of temperature and chamber pressure; also coalescence of micro $\mathrm{NaCl}$ crystals clusters have been detected in SEM micrographs, but more experiments need to be done to be able to conclude this research. As a preliminary conclusion, it can be stablished that $\mathrm{NaCl}$ nanocrystals and microcrystals tend to coalesce in a self-assemble-type configuration following crystallographic directions. Electron microscopy micrographs suggest that nucleation and coalescence occurs at different rates for nano and micro $\mathrm{NaCl}$ crystallites, during film synthesis; the microcrystallites do not growth at the expenses of the small ones. The micrographs presented in Figures1 to 4, correspond to samples deposited in high vacuum.

The results suggest that beside the classics films growth models (Volmer-Weber, Frank-van der Merwe and Stranski-Krastanov) other possibilities must be considered to explain the alkali halides films formation and growth under PLD deposition conditions [4]. 


\section{References:}

[1] R. Eason, Pulsed Laser Deposition of Thin films, Wiley-Interscience, (2007) p. 3-20.

[2] A. Arrieta, S.Mera, R.Diamant, M.Fernández, R. Sosa, L. Escobar, A. Muñoz and E. Haro, Appl. Phys. A 69(7) (1999) S491-S493.

[3] P. E. Dyer, S. M. Maswadi and C.D.Walton, Appl. Phys. A, 76 (2003) 817-822.

[4] The financial support of DGAP-UNAM Project IN 105514 is recognized and appreciated.

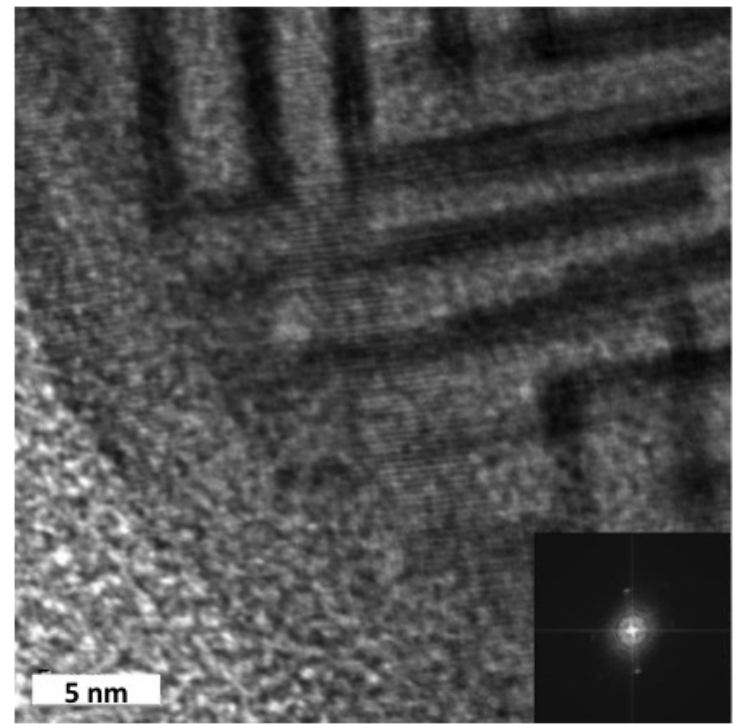

Figure 1. HREM micrograph from a $\mathrm{NaCl}$ nano crystal border: the typical terrace configuration can be observed. The FFT corresponding to a cubic structure is displayed in the lower right corner.

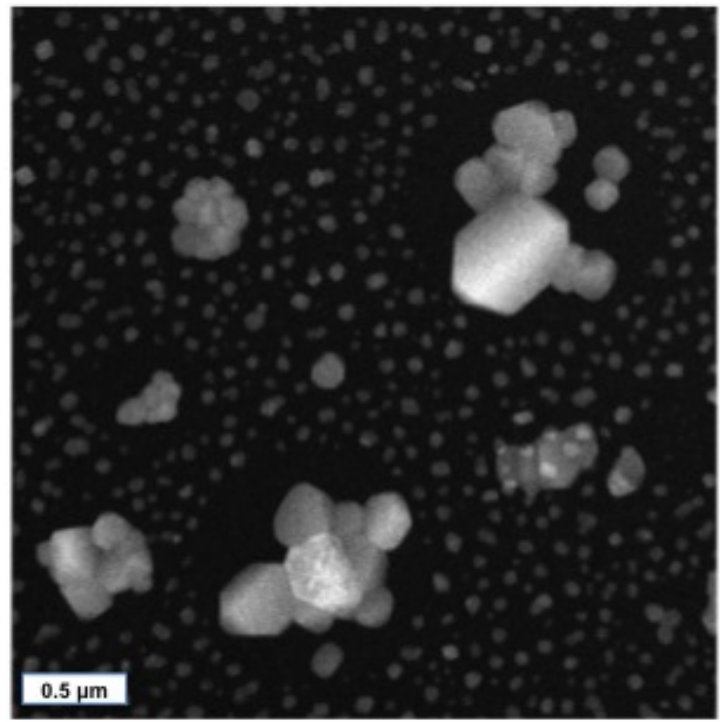

Figure 3. A SEM micrograph showing the coalescence of nano and micro crystals and empty zones surrounding micro $\mathrm{NaCl}$ crystal clusters.

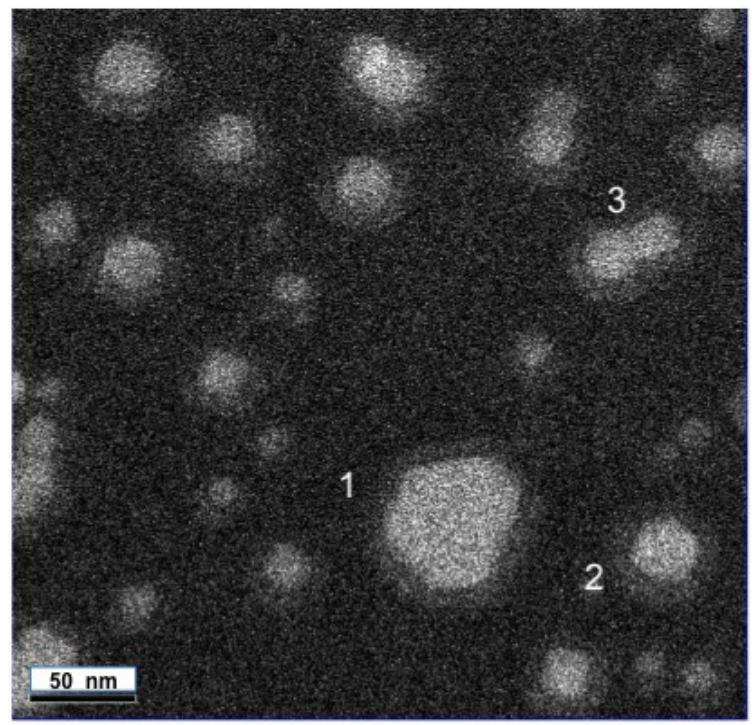

Figure 2. ADF micrograph from a $\mathrm{NaCl}$ sample deposited in high vacuum. A hallo surrounding nano crystallites marked with 1, 2 and 3 and coalescence reveals interesting $\mathrm{NaCl}$ crystals growth details.

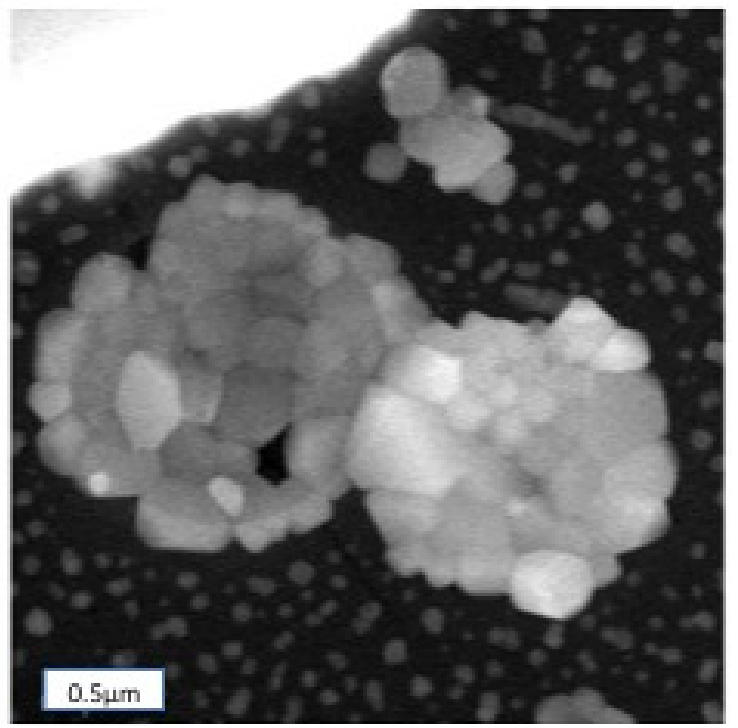

Figure 4. In this SEM micrograph the $\mathrm{NaCl}$ film formation with the coalescence of two micrometric $\mathrm{NaCl}$ crystals clusters can be clearly observed. 